\title{
Modelling the local atomic structure of molybdenum in nuclear waste glasses with $a b$ initio molecular dynamics simulations
}

\author{
Konstantinos Konstantinou, ${ }^{\text {*a }}$ Peter V. Sushko ${ }^{\mathrm{b}}$ and Dorothy M. Duffy ${ }^{\mathrm{a}}$ \\ a Department of Physics and Astronomy, University College London, Gower Street, London, \\ WC1E 6BT, U.K. \\ b Physical Sciences Division, Physical \& Computational Sciences Directorate, Pacific \\ Northwest National Laboratory, Richland, WA, 99352, USA. \\ *E-mail: konstantinos.konstantinou.13@ucl.ac.uk
}

\begin{abstract}
The nature of chemical bonding of molybdenum in high level nuclear waste glasses has been elucidated by ab initio molecular dynamics simulations. Two compositions, $\left(\mathrm{SiO}_{2}\right)_{57.5}$ $-\left(\mathrm{B}_{2} \mathrm{O}_{3}\right)_{10}-\left(\mathrm{Na}_{2} \mathrm{O}\right)_{15}-(\mathrm{CaO})_{15}-\left(\mathrm{MoO}_{3}\right)_{2.5}$ and $\left(\mathrm{SiO}_{2}\right)_{57.3}-\left(\mathrm{B}_{2} \mathrm{O}_{3}\right)_{20}-\left(\mathrm{Na}_{2} \mathrm{O}\right)_{6.8}-\left(\mathrm{Li}_{2} \mathrm{O}\right)_{13.4}-$ $\left(\mathrm{MoO}_{3}\right)_{2.5}$, were considered in order to investigate the effect of ionic and covalent components on the glass structure and the formation of the crystallisation precursors $\left(\mathrm{Na}_{2} \mathrm{MoO}_{4}\right.$ and $\mathrm{CaMoO}_{4}$ ). The coordination environments of Mo cations and the corresponding bond lengths calculated from our model are in excellent agreement with experimental observations. The analysis of the first coordination shell reveals two different types of molybdenum host matrix bonds in the lithium sodium borosilicate glass. Based on the structural data and the bond valence model, we demonstrate that the Mo cation can be found in a redox state and the molybdate tetrahedron can be connected with the borosilicate network in a way that inhibits the formation of crystalline molybdates. These results significantly extend our understanding of bonding in Mo-containing nuclear waste glasses and demonstrate that tailoring the glass composition to specific heavy metal constituents can facilitate incorporation of heavy metals at high concentrations.
\end{abstract}




\section{Introduction}

Mixed alkali and/or alkaline-earth borosilicate glasses are used to immobilise high level nuclear waste through the vitrification process. ${ }^{1,2}$ The additives to pure silica glass enable efficient processing by reducing the glass transition temperature; they also enhance the chemical durability and the waste incorporation capability of the glass matrix. ${ }^{3-5}$

Molybdenum is found in high concentrations in high level nuclear waste and it is known to have low solubility in borosilicate glasses. ${ }^{6}$ For example, $\mathrm{MoO}_{3}$ present in excess of $1 \mathrm{wt} \%$ results in the phase separation of complex molten salts during vitrification, and the formation of the so-called yellow (due to its colour) phase. This phase is associated with formation of crystalline particles, phase-separated from the rest of the molten glass. Yellow phase creates a number of technical and scientific challenges. In particular, (i) yellow phase concentrates at the bottom of the melter and sticks the nozzle of effluent molten glass which accelerates corrosion of the melter; (ii) yellow phase also contains a wide range of fission products and is highly water soluble, which could lead to increased leaching of radioactive nuclei from the vitrified wastes. ${ }^{7}$ The challenge on the scientific side is to identify atomic-scale processes that lead to the formation of molybdate crystals and find approaches to suppressing these processes.

The molybdenum structural environment in inactive nuclear glasses has been investigated using extended X-ray absorption fine structure spectroscopy (EXAFS) ${ }^{8}$ and X-ray absorption near edge structure spectroscopy (XANES) ${ }^{9}$ probing Mo-K edge. The analysis suggests that $\mathrm{Mo}$ tends to form $\left[\mathrm{MoO}_{4}\right]^{2-}$ molybdate groups which are not directly connected to the borosilicate matrix. $\mathrm{Mo}^{6+}$ is the prevailing oxidation state in these borosilicate glass compositions melted in air under oxidising conditions. Molybdate groups are located in depolymerised regions of the glass structure containing cation modifiers (alkali/alkaline-earth). This specific environment of Mo species and its complex structure are thought to be responsible for the low solubility of Mo in the glass network. ${ }^{10}$

The presence of crystalline molybdates may arise from the fact that when nuclear glasses are prepared under oxidising $\left(\mathrm{O}_{2}\right)$ or neutral (air atmosphere) conditions, molybdenum mainly occurs in the oxidation state $\mathrm{Mo}^{6+}$. However, other oxidation states, such as $\mathrm{Mo}^{3+}, \mathrm{Mo}^{4+}$ and $\mathrm{Mo}^{5+}$, may be present under more reducing conditions or appear transiently as a result of the concentration fluctuations at the processing conditions. These species would inevitably create other structural elements, which 
would have different types of bonding to the glass network. Different oxidation states, known as redox states in commercial bulk glass compositions have been studied before, ${ }^{11}$ however, the effects of redox states on nuclear waste glasses have received much less attention.

The formation of the yellow phase is highly undesirable, therefore, it is imperative to suppress the crystallisation of the molybates during melt cooling. It is expected that the segregation rate of these crystalline phases and the solubility of molybdenum can be influenced by the chemical composition of the glass host. For instance, MAS NMR and Raman spectroscopy studies ${ }^{10,12}$ revealed that when $\mathrm{B}_{2} \mathrm{O}_{3}$ concentration increases, $\mathrm{Na}$ cations move from their sites near non-bridging oxygen atoms to preferentially bind to $\mathrm{BO}_{4}$ tetrahedra. This modification of the distribution of the $\mathrm{Na}$ cations within the glass led to a modification in the nature of molybdate crystals formed during melt cooling (the volume fraction of $\mathrm{CaMoO}_{4}$ became larger while the volume fraction of $\mathrm{Na}_{2} \mathrm{MoO}_{4}$ became smaller). It is crucial to design glasses in which the formation of these molybdate phases is suppressed in order to maximise the waste disposal efficiency.

Molecular dynamics (MD) is a computational method of choice for efficiently probing the various heterogeneous local environments found in glasses, as it provides insight into the material properties from the atomistic level. ${ }^{13}$ However, classical MD simulations are hampered by the lack of reliable force fields to describe the complex interactions in multicomponent borosilicate glasses, ${ }^{14}$ even though reliable interatomic potentials have been developed to model pure silica and mixed cation silicate glasses. ${ }^{15}$ An alternative computational approach is the ab initio molecular dynamics technique (AIMD), a parameter free approach, where the forces are computed from a quantum mechanical representation of the electronic structure. Despite being computationally demanding, as compared to classical MD simulations, this approach enables accurate modelling of many-body systems, and it can account for switching chemical bonds and electron density polarisation. AIMD simulations have previously been used to model the structural properties of pure silica and alkali silicate glasses, ${ }^{16-}$ ${ }^{20}$ as well as, sodium borosilicate glass ${ }^{21}$ and bioactive phosphate glasses. ${ }^{22-25}$

In this study, ab initio Born-Oppenheimer molecular dynamics simulations were performed in order to model the local atomic structure of molybdenum in lithium sodium and calcium sodium borosilicate glasses. In particular, we focus on the structural effects determined by the inclusion of the molybdenum cations in the glass structure and how they are embedded into the network. The structural motifs of the molybdenum shortrange ordering in our simulated glass structures will allow us to gain direct insight on the yellow phase formation and evaluate the solubility of molybdenum in nuclear waste glasses. 


\section{Methods}

Two systems with composition $\left(\mathrm{SiO}_{2}\right)_{57.5}-\left(\mathrm{B}_{2} \mathrm{O}_{3}\right)_{10}-\left(\mathrm{Na}_{2} \mathrm{O}\right)_{15}-(\mathrm{CaO})_{15}-$ $\left(\mathrm{MoO}_{3}\right)_{2.5}$ and $\left(\mathrm{SiO}_{2}\right)_{57.3}-\left(\mathrm{B}_{2} \mathrm{O}_{3}\right)_{20}-\left(\mathrm{Na}_{2} \mathrm{O}\right)_{6.8}-\left(\mathrm{Li}_{2} \mathrm{O}\right)_{13.4}-\left(\mathrm{MoO}_{3}\right)_{2.5}$ (thereafter referred as MCNB and MLNB respectively) were modelled using periodic boundary conditions. The molar composition of the MCNB glass is based on nuclear waste glass compositions that have been previously studied experimentally..$^{12}$ In order to investigate the dependence of Mo local atomic structure and its stability within the borosilicate glass network on the chemical nature and composition of the glass host, we increased the molar composition of $\mathrm{B}_{2} \mathrm{O}_{3}$, decreased the concentration of $\mathrm{Na}_{2} \mathrm{O}$ and substituted the $\mathrm{CaO}$ with $\mathrm{Li}_{2} \mathrm{O}$ keeping its concentration at similar level, resulting in the MLNB composition. The concentration of $\mathrm{MoO}_{3}$ was the same in both glasses in order to avoid effects due to compositional dependence of the molybdenum.

The starting configurations were generated by placing atoms randomly in a cubic simulation box with imposed constraints to avoid un-physically small interatomic distances. The total number of atoms for the MCNB glass was 202 (38 Si, $12 \mathrm{~B}, 20 \mathrm{Na}$, $10 \mathrm{Ca}, 2 \mathrm{Mo}$ and $120 \mathrm{O}$ ), while for the MLNB glass it was 226 (38 Si, $26 \mathrm{~B}, 8 \mathrm{Na}, 18 \mathrm{Li}$, $2 \mathrm{Mo}$ and $134 \mathrm{O}$ ). The simulation cell sizes, $13.61 \AA$ and $13.90 \AA \AA$ for MCNB and MLNB respectively, were chosen to give the appropriate density and kept constant throughout the simulation.

The density was calculated for the alkali alkaline-earth and mixed alkali borosilicate glasses before the addition of the $\mathrm{MoO}_{3}$. Classical MD simulations with the DL_POLY classic package,${ }^{26}$ using a pairwise Lennard - Jones potential mode ${ }^{27-29}$ and a melt-and-quench approach, were performed in order to generate the amorphous structures. The NPT ensemble (constant number of particles, pressure and temperature), using a Nosé-Hoover thermostat and barostat, ${ }^{30-32}$ was applied to calculate the density of each structure at $300 \mathrm{~K}$ and 0 bar. The accuracy of the calculated density was subsequently increased by running AIMD simulations at $300 \mathrm{~K}$ with the NPT ensemble and a CSVR thermostat and barostat. ${ }^{33}$ The calculated density for the calcium sodium borosilicate glass structure $\left(2.59 \mathrm{~g} / \mathrm{cm}^{3}\right)$ is in very good agreement with the experimental value $\left(2.62 \mathrm{~g} / \mathrm{cm}^{3}\right) .{ }^{34}$ There are no experimental density measurements available for the exact composition of the simulated mixed alkali borosilicate glass, however, the calculated density of this glass composition (2.49 $\left.\mathrm{g} / \mathrm{cm}^{3}\right)$ is in good agreement with experimental densities $\left(2.43-2.47 \mathrm{~g} / \mathrm{cm}^{3}\right)$ for lithium sodium borosilicate glasses of similar molar composition. ${ }^{35}$

The densities of the Mo-containing nuclear waste glasses modelled in this study were not obtainable experimentally, nor available via glass property modelling databases. We have 
therefore estimated the effect on the density of incorporating Mo to the two glass compositions performing a cell optimisation using variable cell AIMD with 1.0 fs timestep and a convergence threshold of $0.1 \mathrm{kbar}$ for the components of the stress tensor.

Born-Oppenheimer AIMD simulations were carried out using the CP2K code. ${ }^{36}$ The electronic structure was treated through the Kohn-Sham formulation of density functional theory (DFT) ${ }^{37}$ using the generalised gradient approximation (GGA) with the Perdew-Burke-Ernzerhof (PBE) exchange-correlation functionals. ${ }^{38}$ The Gaussian basis set employed for all the atomic species was a double- $\zeta$ basis set with polarisation functions $(\mathrm{DVZP})^{39}$ in conjunction with the Goedecker-Teter-Hutter (GTH) pseudopotential. ${ }^{40}$ The plane-wave energy cutoff was 700 Ry and the MD timestep was 2.0 fs.

The glass structures were generated using a melt-and-quench approach. The canonical ensemble (constant number of particles, volume and temperature or NVT) was applied and the Nosé-Hoover thermostat chain, ${ }^{30-32}$ with a relaxation constant 0.1 ps, was chosen to control the temperature fluctuations. For each composition, the initial configuration was heated up at $2300 \mathrm{~K}$ with a $25 \mathrm{ps}$ AIMD run to ensure that the system was melted and well equilibrated at this temperature. Despite a small drift in the total energy the recorded energy fluctuations were lower than $0.001 \%$. The molten structure was subsequently cooled using a stepwise process, consisting of a series of nine NVT AIMD runs of 10 ps each, with target temperatures set to $2000 \mathrm{~K}, 1800 \mathrm{~K}, 1600 \mathrm{~K}, 1400$ $\mathrm{K}, 1200 \mathrm{~K}, 1000 \mathrm{~K}, 800 \mathrm{~K}, 600 \mathrm{~K}$ and $300 \mathrm{~K}$. At $300 \mathrm{~K}$ the structure was further equilibrated for $10 \mathrm{ps}$, followed by a final AIMD production run of $10 \mathrm{ps}$, to collect the structural data. This computational scheme corresponds to a total simulation time of $135 \mathrm{ps}$ and a nominal cooling rate of around $20 \mathrm{~K} / \mathrm{ps}$. Cooling rates of this order of magnitude have been used in previous simulation studies, using AIMD, ${ }^{21,23-25,41,42}$ in order to prepare accurate structural models of glasses that are in agreement with experimental results.

We note that, the fixed volume approach for the melt-and-quench process will generate glass structure with high final pressure in the cell. However, this residual pressure does not affect the quality of the calculations, as relaxation of the cell results in almost no difference in the final glass volume for both compositions (see Tables S1 and S2, ESI ). 


\section{Results and discussion}

Silicon and boron atoms have the role of the network formers in the continuous random glass network. ${ }^{43}$ The local environment around Si and $\mathrm{B}$ was analysed through the pairwise radial distribution function, $g(r)$, shown in Fig. 1a for the MLNB glass. In both glass compositions, Si was found to be $100 \%$ 4-coordinated to oxygen atoms with an average $\mathrm{Si}-\mathrm{O}$ interatomic distance of $1.64 \AA$. The $\mathrm{O}-\mathrm{Si}-\mathrm{O}$ bond angle distribution, $g(\theta)$, shown in Fig. 1b, provides further details about the first coordination shell of $\mathrm{Si}$ atoms in the glass structure. The peak of the distribution is located at $108.5^{\circ}$, close to the tetrahedral angle $\left(109.5^{\circ}\right)$, which is indicative of the formation of near perfect $\mathrm{SiO}_{4}$ tetrahedra in the simulated glass structure.

The average B - O interatomic distance is $1.40 \AA$ and $1.41 \AA$ for the MCNB and MLNB glasses respectively, while the average coordination number is 3.2 and 3.5 for the MCNB and MLNB glasses respectively. Further analysis of the glass models shows that $B$ ions have two different coordination environments within each glass structure. 3 and 4-coordinated $\mathrm{B}$ ions were found to exist simultaneously in both glasses in the form of $\mathrm{BO}_{3}$ triangles and $\mathrm{BO}_{4}$ tetrahedra respectively. The $\mathrm{O}-\mathrm{B}-\mathrm{O}$ bond angle distribution, $g(\theta)$, shown in Fig. $1 \mathrm{~b}$ for both compositions, provides further details about the structure of the $B$ environment in the two glass models. In MCNB glass, the $\mathrm{O}-\mathrm{B}-\mathrm{O} \mathrm{g}(\theta)$ reveals two almost equivalent peaks at $110.5^{\circ}$ and $121.5^{\circ}$ which, along with the average coordination number of 3.2 indicate that $B$ ions have flexible local environment within the MCNB glass forming triangles and tetrahedra. In MLNB glass the O-B-O $g(\theta)$ has one peak at $110.5^{\circ}$ which suggests that $B$ ions have a stronger preference for tetrahedral local environment in the lithium sodium borosilicate (MLNB) glass model. However, the large width of the $g(\theta)$ in MLNB glass, along with the coordination number of 3.5 , indicate that the system contains also distorted, i.e., non-planar 3-coordinated $B$ ions.

The typical measurements from the literature for the $\mathrm{Si}-\mathrm{O}$ bond length are 1.58 - $1.64 \AA$ for a broad range of silicate glasses. ${ }^{44-46}$ Cormier et al ${ }^{47}$ reported a $\mathrm{Si}-\mathrm{O}$ interatomic distance of $1.60 \AA$ in borosilicate glasses. The $\mathrm{B}-\mathrm{O}$ bond length was measured by Majérus et al ${ }^{48} 1.38 \AA$ and $1.47 \AA$ for 3 - and 4-coordinated $B$ atoms respectively. Moreover, our calculated results are in very good agreement with previous modelling study in lithium sodium borosilicate glasses, as Connely et al ${ }^{49}$ reported for $\mathrm{Si}$ and $\mathrm{B}$ similar interatomic distances $(1.58 \AA$ and $1.41 \AA$ for $\mathrm{Si}-\mathrm{O}$ and $\mathrm{B}-\mathrm{O}$ respectively) and coordination environments (4 and 3.5 for Si and $B$ respectively). 

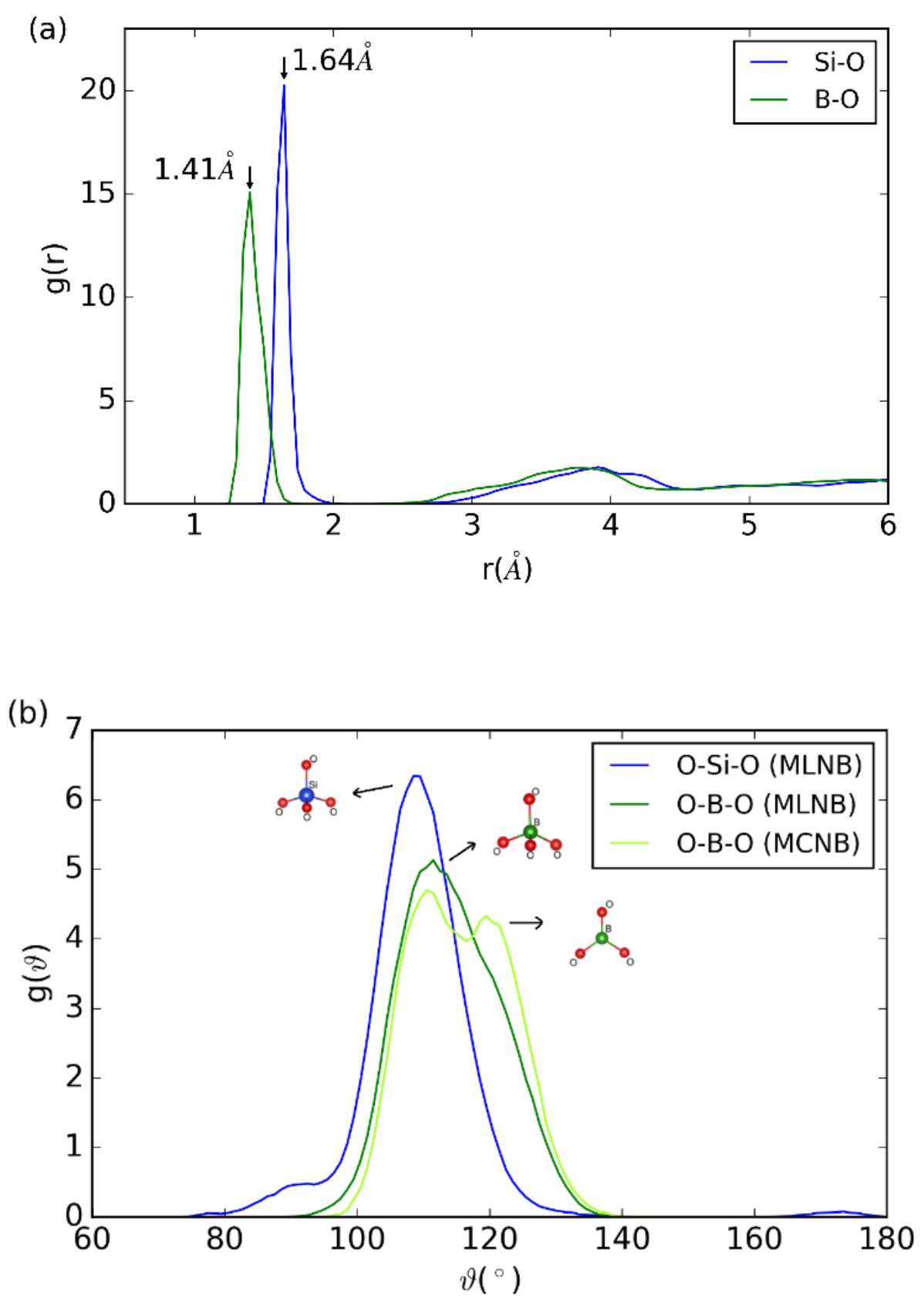

Fig. 1 (a) Pairwise radial distribution function for network former ( $\mathrm{Si}$ and $\mathrm{B}$ atoms) - oxygen interatomic distance in the simulated lithium sodium borosilicate nuclear glass (MLNB composition) and (b) oxygensilicon-oxygen bond angle distribution in MLNB glass and oxygen-boron-oxygen bond angle distribution in MLNB and MCNB compositions. Tetrahedral geometry for $\mathrm{Si}$ in its first coordination shell within the MLNB glass. Boron is present in planar $\left(g(\theta) \sim 120^{\circ}\right)$ and tetrahedral $\left(g(\theta) \sim 109.5^{\circ}\right)$ environments within both glass structures. The $g(r)$ functions, as well as, the $\mathrm{O}-\mathrm{Si}-\mathrm{O} g(\theta)$ are indistinguishable for the MLNB and MCNB compositions. 
Structural analysis for the short-range ordering of the alkali and alkaline-earth cations has also been performed through the pairwise radial distribution functions, $g(r)$, shown in Fig. 2a. The average bond lengths for the three cations are $\mathrm{Ca}-\mathrm{O}=2.30 \AA$, $\mathrm{Li}-\mathrm{O}=1.94 \AA$ and $\mathrm{Na}-\mathrm{O}=2.34 \AA$ and $2.39 \AA$ for the MCNB and MLNB glasses respectively. The average oxygen coordination number around $\mathrm{Ca}$, $\mathrm{Li}$ and $\mathrm{Na}$ was found to be 5.8, 4.1 and 5.6 (in MCNB) - 6.7 (in MLNB) respectively.

A previous modelling study in lithium sodium borosilicate glasses ${ }^{49}$ reported an average $\mathrm{Li}$ - O interatomic distance $2.07 \AA$ with an average coordination number 3.5, while a neutron diffraction study in lithium silicate glasses ${ }^{50}$ reported 4-coordinated Li atoms and an $\mathrm{Li}$ - O interatomic distance of $1.99 \AA$. The $\mathrm{Na}-\mathrm{O}$ bond length was calculated in borosilicate glasses $2.2-2.55 \AA$ by Cormier et al ${ }^{47}$ and $2.82 \AA$ by Connelly et $a{ }^{49}$ with an average coordination number 8 in both studies. Michel et al ${ }^{34}$ calculated the $\mathrm{Ca}$ - O interatomic distance $2.35 \AA$ in nuclear glass compositions.

The distributions of $\mathrm{O}-$ cation-O angles, $g(\theta)$, shown in Fig. $2 b$ for the two simulated glass compositions, further highlight the structure of the coordination shell of the three cations and give information about the connection of the cations with the oxygen species within the glass structures. A peak in the $\mathrm{g}(\theta)$ at $\sim 90^{\circ}$ is due to the bonding between the cation and two non-bridging oxygen atoms (NBO-cation-NBO) belonging to different chains of tetrahedra in the borosilicate network. A peak in the $g(\theta)$ at $\sim 60^{\circ}$ originates from the linkage of the cation to two bridging oxygen atoms (BO-cation-BO) or one bridging and one non-bridging oxygen (BO-cation-NBO) belonging to the same tetrahedron.

In $\mathrm{O}-\mathrm{Ca}-\mathrm{O} g(\theta)$ function there is one main peak located at $83.5^{\circ}$. This indicates that the Ca distribution is dominated by the NBO-Ca-NBO contribution. The combined information of the average coordination number ( 6.0) together with the $g(\theta)\left(\sim 90^{\circ}\right)$ suggests that the first coordination shell of the cation has a strong preference for the octahedral geometry. The $\mathrm{O}-\mathrm{Li}-\mathrm{O} g(\theta)$ function feature two peaks located at $68.5^{\circ}$ and $106.5^{\circ}$. The intensity of the second peak is stronger than that of the first peak and is quite close to the tetrahedral value. This, together with the 4-coordinated Li cations suggests that there are $\mathrm{LiO}_{4}$ tetrahedra in the MLNB glass structure.

There are also two peaks in the $\mathrm{O}-\mathrm{Na}-\mathrm{O} \mathrm{g}(\theta)$ functions located at $58.5^{\circ} / 86.5^{\circ}$ and $57.5^{\circ} / 99.5^{\circ}$ for the MCNB and MLNB glasses respectively. The Na cations tend to form octahedra in both glass structures, but the connection of the cation with the oxygen species changes. The intensity of the first peak for the $g(\theta)$ function in the MLNB glass is stronger than that of the respective peak in the MCNB glass. Na cations shows a stronger preference to connect with two bridging oxygen atoms or one bridging and one non-bridging oxygen of the same $\mathrm{SiO}_{4}$ and/or $\mathrm{BO}_{4}$ tetrahedron within the MLNB glass. 
(a)

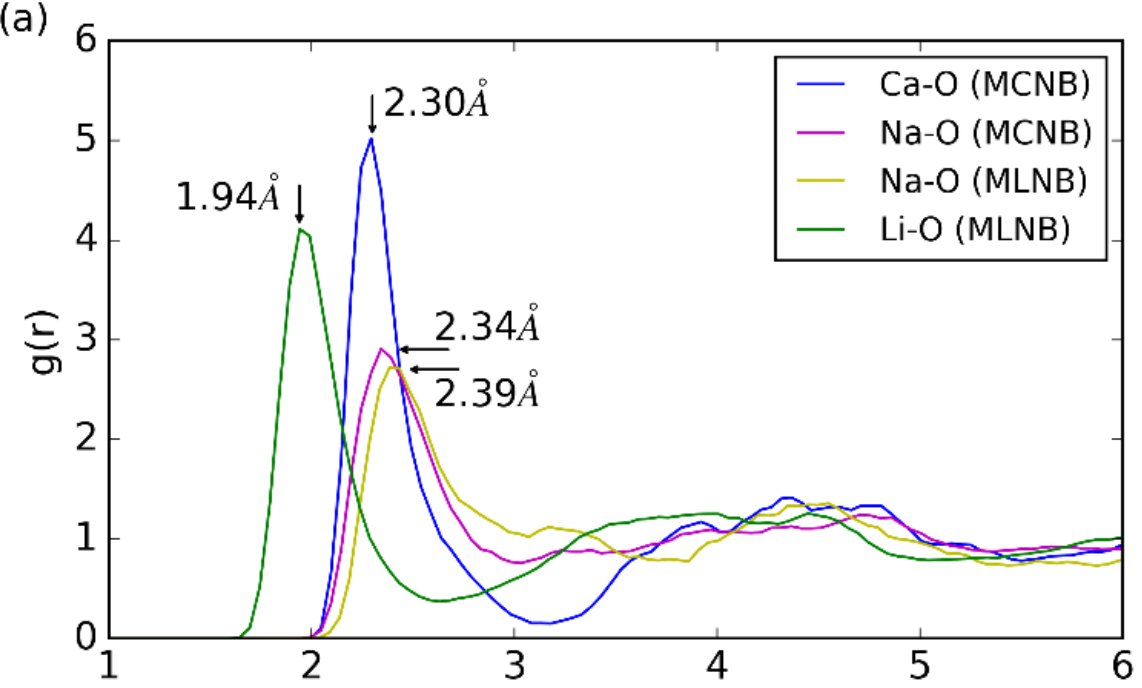

$\mathrm{r}(\AA)$

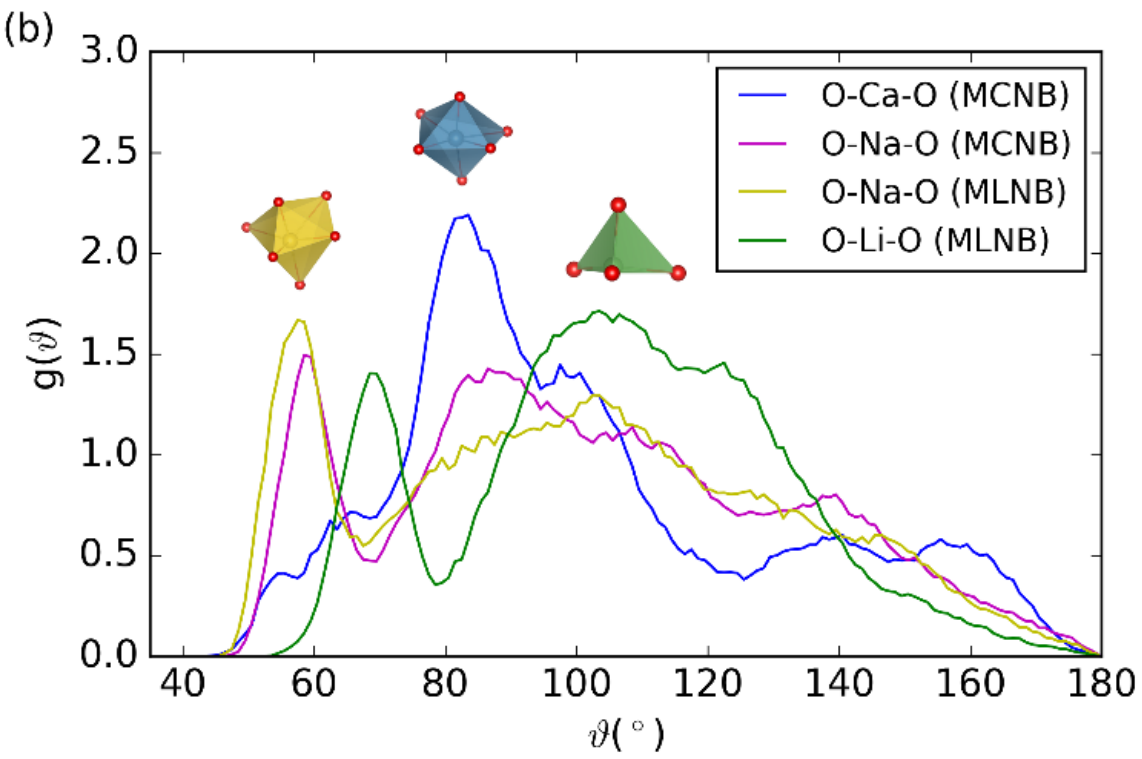

Fig. 2 (a) Pairwise radial distribution functions for the alkali ( $\mathrm{Li}$ and $\mathrm{Na}$ cations) - and alkalineearth (Ca cation) - oxygen interatomic distances and (b) oxygen-cation-oxygen bond angle distributions in the MCNB and MLNB simulated glass structures. Octahedral geometry for $\mathrm{Na}$ and $\mathrm{Ca}$ cations and tetrahedral geometry for $\mathrm{Li}$ cations in their first coordination shell. Yellow ball is $\mathrm{Na}$, light blue ball is $\mathrm{Ca}$, light green ball is $\mathrm{Li}$ and red ball is $\mathrm{O}$.

The bond lengths and coordination numbers for $\mathrm{Si}, \mathrm{B}, \mathrm{Ca}, \mathrm{Li}$ and $\mathrm{Na}$ in the MCNB and MLNB simulated glasses are summarised in Tables 1 and 2. 
Table 1 Average oxygen interatomic distances for the network formers ( $\mathrm{Si}$ and $\mathrm{B}$ ) and the alkali ( $\mathrm{Na}$ and $\mathrm{Li}$ ) and alkaline-earth $(\mathrm{Ca})$ cations in the two simulated glass structures. The bond lengths correspond to the peak position of the respective pairwise radial distributions.

\begin{tabular}{lll}
\hline & \multicolumn{2}{l}{ Bond length $(\AA)$} \\
\cline { 2 - 3 } Atom pair & MCNB & MLNB \\
\hline $\mathrm{Si}-\mathrm{O}$ & 1.64 & 1.64 \\
$\mathrm{~B}-\mathrm{O}$ & 1.40 & 1.41 \\
$\mathrm{Na}-\mathrm{O}$ & 2.34 & 2.39 \\
$\mathrm{Ca}-\mathrm{O}$ & 2.30 & - \\
$\mathrm{Li}-\mathrm{O}$ & - & 1.94 \\
\hline
\end{tabular}

Table 2 Average oxygen coordination numbers for the network formers ( $\mathrm{Si}$ and $\mathrm{B}$ ) and the alkali ( $\mathrm{Na}$ and $\mathrm{Li}$ ) and alkaline-earth $(\mathrm{Ca})$ cations in the two simulated glass structures. The coordination numbers were calculated from the integration of the first peak in the respective pairwise radial distributions.

\begin{tabular}{lll}
\hline \multirow{2}{*}{ Atom } & \multicolumn{2}{l}{ Coordination number } \\
\cline { 2 - 3 } & MCNB & MLNB \\
\hline $\mathrm{Si}$ & 4 & 4 \\
$\mathrm{~B}$ & 3.2 & 3.5 \\
$\mathrm{Na}$ & 5.6 & 6.7 \\
$\mathrm{Ca}$ & 5.8 & - \\
$\mathrm{Li}$ & - & 4.1 \\
\hline
\end{tabular}

The local environment around the Mo cations was characterised using the pairwise radial distribution function, $g_{M o-O}(r)$, shown in Fig. 3 . The first peak of the distribution function gives information about the first coordination shell of the Mo cations in the simulated nuclear glasses. For MCNB glass the $g(r)$ has one clear peak which corresponds to an average bond length $\mathrm{Mo}-\mathrm{O}=1.75 \AA$, whereas for MLNB glass the $g(r)$ has two distinct peaks in the first coordination shell. The strongest peak corresponds to a Mo - O bond length of $1.75 \AA$, as in the MCNB glass structure, and 
the other sharp peak corresponds to a Mo - O bond length of $1.94 \AA$. The average oxygen coordination number around Mo cations was found to be 4.0 and 4.2 for the MCNB and MLNB glasses respectively, using the same cutoff for the integration of the first peak in $g(r)$. Thus, the molybdenum ions are mainly 4-fold coordinated to oxygen atoms in our simulated glass structures. We note that, we have used two different values for the cutoff, one corresponding to the local minima (2.2 $\AA$ ) and the other corresponding to the value $g(r)$ goes to zero $(2.7 \AA)$ and we observed no significant differences in the calculated coordination numbers for both compositions.

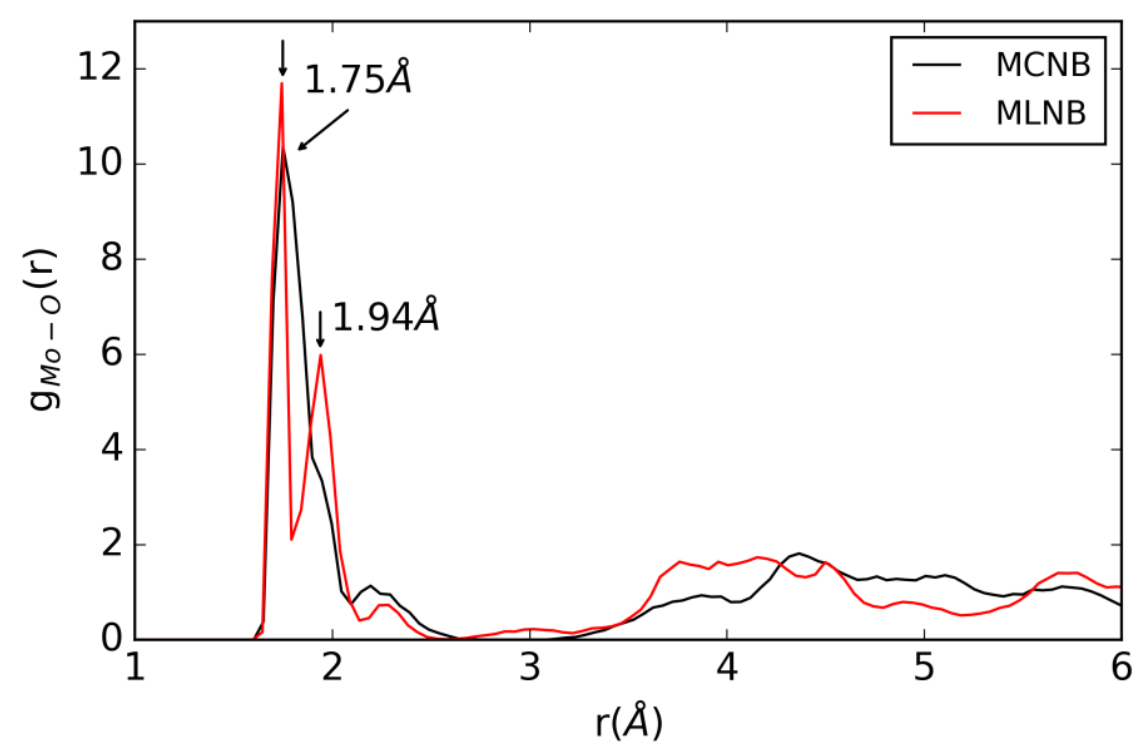

Fig. 3 Pairwise radial distribution function for molybdenum - oxygen interatomic distance in the MCNB and MLNB simulated glass structures. The maxima at $1.75 \AA$ coincide for the two glass compositions.

The $g(\theta)$ can provide further information about the structure of the coordination shell of the Mo cations. The O-Mo-O $g(\theta)$ function for the MLNB composition is illustrated in Fig. 4. It can be seen that there is one main peak located at $102.5^{\circ}$ which has the higher intensity in the distribution and is close to the value of the perfect tetrahedron $\left(109.5^{\circ}\right)$. The combined information of the average oxygen coordination number and the $g(\theta)$ indicates that the coordination environment of Mo cations in our simulated nuclear glasses has a strong preference to tetrahedral geometry, resulting in the formation of $\mathrm{MoO}_{4}$ molybdate groups. 


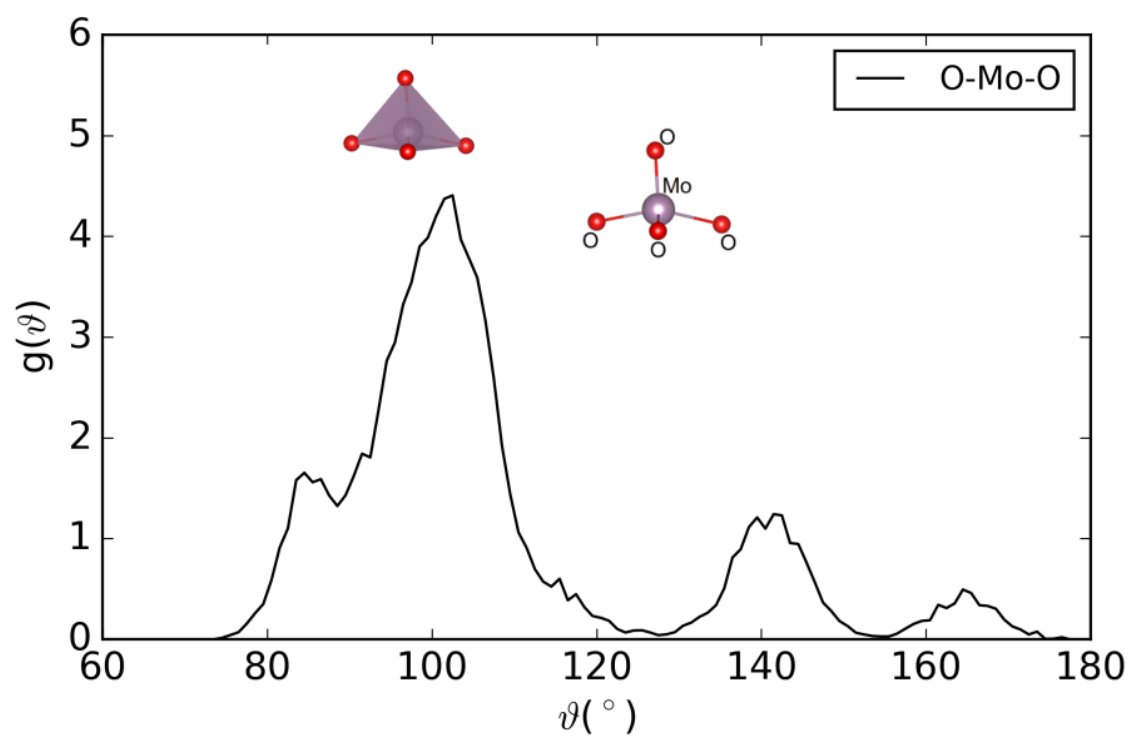

Fig. 4 Oxygen-Molybdenum-Oxygen bond angle distribution in the MLNB simulated glass structure. The distribution shows three peaks and one shoulder. The main peak corresponds well to the tetrahedral coordination, in agreement with experimental data. ${ }^{8}$

Table 3 summarises the values of bond length, coordination number and bond angle for the Mo cation in the two simulated glass structures. Our simulated results are in very good agreement with EXAFS and XANES studies in nuclear borosilicate glasses. ${ }^{7,9,10}$ The EXAFS data acquired from high level nuclear waste glasses with similar compositions show an analogous picture for the molybdenum local environment. ${ }^{8}$ These data demonstrate that Mo cations are 4-coordinated to oxygen with an average Mo - O interatomic distance of $1.76-1.78 \AA$, which leads to the formation of $\mathrm{MoO}_{4}$ tetrahedrally coordinated species.

Table 3 Analysis of the Mo first coordination shell, through the bond length, coordination number and bond angle in the two simulated glass structures. We used the same cutoff for the integration of the first peak in the $g(r)$ of each glass.

\begin{tabular}{llll}
\hline Composition & Mo $-\mathrm{O}(\dot{\mathrm{A}})$ & $\begin{array}{l}\text { Coordination } \\
\text { number }\end{array}$ & $\mathrm{O}-\mathrm{Mo}-\mathrm{O}\left(^{\circ}\right)$ \\
\hline MCNB & 1.75 & 4 & 103.5 \\
MLNB & $1.75 \& 1.94$ & 4.2 & 102.5 \\
\hline
\end{tabular}


We note that, due to the small number of Mo cations in the periodic cell, the amount of data is insufficient for accurate statistical representation of the local atomic structure of Mo. Nevertheless, these results are sufficient in order to discern between different types of local structures. In addition, the results of the simulations are in good agreement with experimental data which gives us confidence about the accuracy of our model. Therefore, we treat these structures as representative for the purpose of this study. We also note that, as AIMD is computationally very expensive, the available computational resources limit the size of our glass models.

The $\mathrm{MoO}_{4}$ tetrahedral formation within the glass structure, and also its connection with the other species, for the MCNB and MLNB glasses, are shown in Fig. 5 and Fig. 6. In the MCNB glass the molybdate unit is located in a region surrounded by modifier cations ( $\mathrm{Na}$ and $\mathrm{Ca}$ ) and it is not linked directly to the borosilicate network. This specific structural position within cationic domains and the absence of connectivity with the polymerised network explains the low solubility of Mo in nuclear waste glasses and the possible phase separation of a yellow phase consisting of sodium or calcium molybdates.
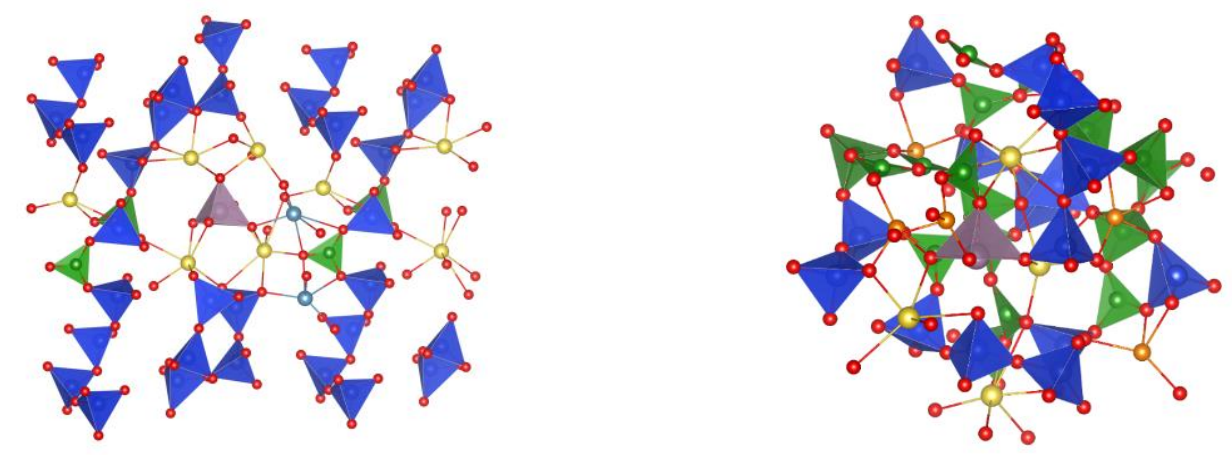

Fig. 5 Tetrahedral local environment of the Mo cation and location within the glass network in the MCNB (top) and MLNB (bottom) simulated nuclear glass structures. In the MCNB glass the $\mathrm{MoO}_{4}$ tetrahedron is linked to the rest of the host lattice via the ionic bonds only. In the MLNB glass the $\mathrm{MoO}_{4}$ unit is located closer to the borosilicate network and there is connection with the network formers. Light gray ball is $\mathrm{Mo}$, blue ball is $\mathrm{Si}$, green ball is $\mathrm{B}$, yellow ball is $\mathrm{Na}$, light blue ball is $\mathrm{Ca}$, orange ball is $\mathrm{Li}$ and red ball is $\mathrm{O}$. 


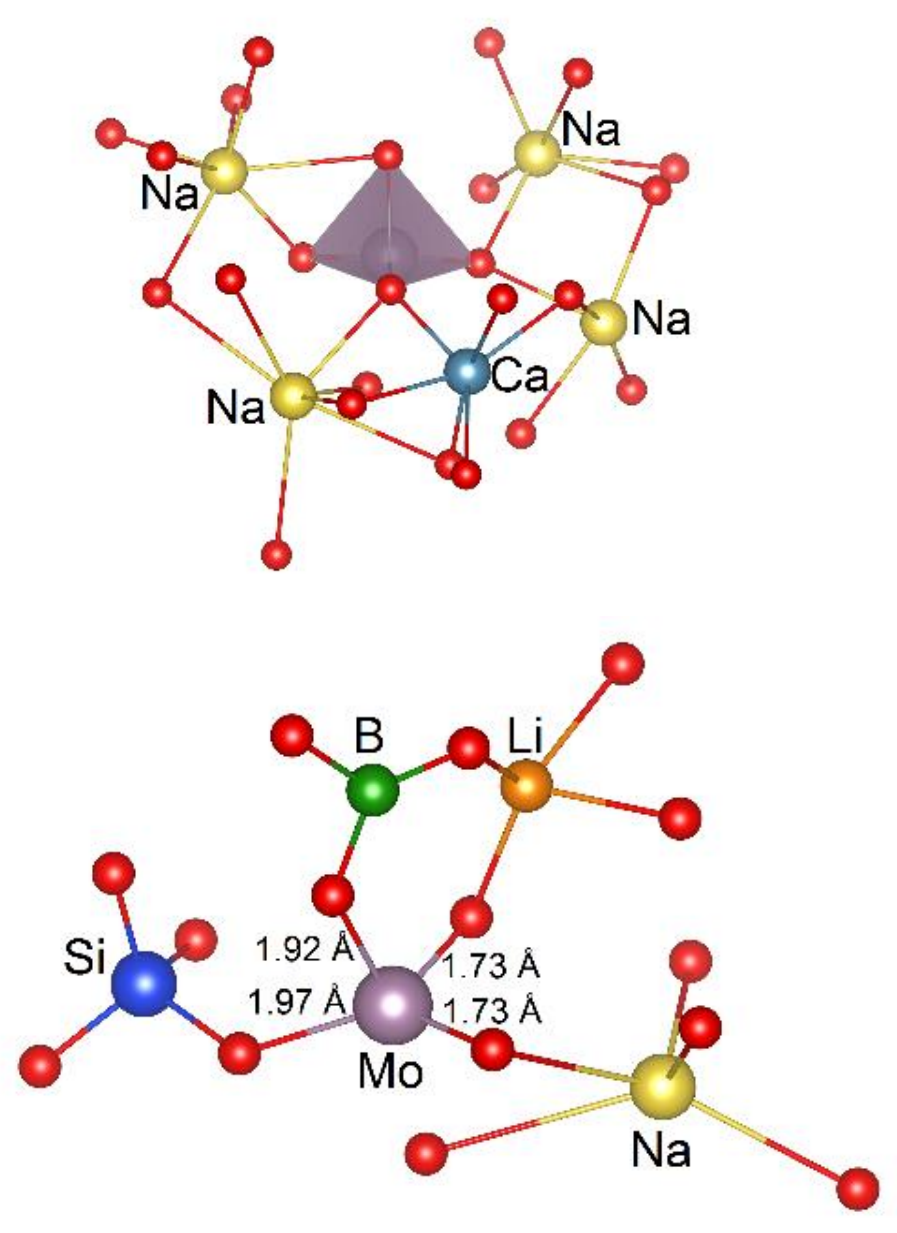

Fig. 6 Tetrahedral coordination environment of the Mo cation and connection with the other species in the MCNB (top) and MLNB (bottom) simulated nuclear glasses. In the MCNB glass the $\mathrm{MoO}_{4}$ tetrahedron is surrounded by $\mathrm{Na}$ and $\mathrm{Ca}$ cations and there is no connectivity with the borosilicate network, whereas in the MLNB glass there is partial connection of the molybdate unit with the polymerised network of the glass due to the longer Mo - $\mathrm{O}$ bonds. Light gray ball is Mo, blue ball is $\mathrm{Si}$, green ball is $\mathrm{B}$, light blue ball is $\mathrm{Ca}$, orange ball is $\mathrm{Li}$, yellow ball is $\mathrm{Na}$ and small red ball is $O$.

In the MLNB glass (bottom panel in figures 5 and 6), for the 4-coordinated Mo cation, two of the Mo - $\mathrm{O}$ bonds are due to the first peak in the $\mathrm{g}(\mathrm{r})$, which corresponds to a shorter interatomic distance, whereas the two other bonds correspond to the second peak at the slightly larger interatomic distance (see Fig. 3). The longer $\mathrm{Mo}-\mathrm{O}$ bond lengths enable the $\mathrm{MoO}_{4}$ tetrahedron to connect with $\mathrm{Si}$ and $\mathrm{B}$ atoms and incorporate into the borosilicate glass network. This picture confirms that for average Mo - O interatomic distances $\approx 1.75-1.78 \AA$, the molybdate tetrahedra do not link to network formers. 
The bond valence model ${ }^{51}$ has been used in order to further investigate the molybdenum oxygen linkages with the network formers and network modifiers in our simulated glass structures. The valence, $\mathrm{S}$, of the $\mathrm{Mo}-\mathrm{O}$ bond was calculated using the equation:

$$
S_{\mathrm{Mo}-\mathrm{O}}=\exp \left[\frac{R_{0}-d(\mathrm{Mo}-\mathrm{O})}{b}\right]
$$

where $R_{0}=1.907 \AA$ is the bond valence parameter for an oxidation state of $\mathrm{Mo}^{6+}, \mathrm{d}(\mathrm{Mo}-\mathrm{O})$ is the molybdenum oxygen interatomic distance and $b$ is a constant $(b=0.37 \AA) .{ }^{52,53}$ According to the model, the sum of the bond valences relative to an oxygen atom must be close to the theoretical value of 2.0 valence units.

The $\mathrm{Mo}-\mathrm{O}-\mathrm{Si}$ linkage in Fig. 6 corresponds to $\mathrm{Mo}-\mathrm{O}=1.97 \AA$ and, based on equation (1), the bond valence is $S_{\text {Mo-O }}=0.84 \mathrm{v}$.u.. The sum of the bond valences for the particular oxygen is $\mathrm{S}_{\mathrm{Mo}-\mathrm{O}}+\mathrm{S}_{\mathrm{Si}-\mathrm{O}}=1.84$ v.u. $<2.0$ v.u., assuming that for the 4 -coordinated $\mathrm{Si}$ atom the bond valence is $\mathrm{S}_{\mathrm{Si}-\mathrm{O}}=1.0$ v.u. ${ }^{54}$ The Mo $-\mathrm{O}-\mathrm{B}$ linkage in Fig. 6 corresponds to $\mathrm{Mo}-\mathrm{O}=1.92 \AA$ and the relative bond valences are $S_{\mathrm{Mo}-\mathrm{O}}=0.98 \mathrm{v}$.u. and $\mathrm{S}_{\mathrm{B}-\mathrm{O}}=0.77 \mathrm{v} . \mathrm{u}$. for the 3-coordinated $\mathrm{B}$ atom, ${ }^{54}$ which results in a sum of the bond valences 1.75 v.u. $<2.0$ v.u.. In both cases, the Pauling's stability rules ${ }^{55}$ are fulfilled, as the sum of the bond valences is less than $2.0 \mathrm{v} . \mathrm{u}$. for the oxygen between Mo and Si or B respectively, which means that the specific oxygen atom will not be over-bonded. Consequently, the linkages with the network formers are not forbidden within the glass network for the larger Mo - O interatomic distances, which are possible in the first coordination environment of Mo cations in the MLNB glass.

For the two shorter Mo - O bonds (1.73 $\AA$ ) in Fig. 6 the calculated bond valence is SMo-O $=1.61$ v.u. . The molybdenum oxygen linkages with $\mathrm{Si}$ and $\mathrm{B}$ in these cases are forbidden as the sum of the bond valences would be 2.61 and $2.38 \mathrm{v}$.u. respectively, which are much larger than $2.0 \mathrm{v}$.u. for the oxygen between the cations. In such a situation the oxygen atom would be overbonded, so for these shorter interatomic distances the oxygen from the Mo - O bond is connected to an alkali cation.

In MCNB glass the calculated, $\mathrm{S}_{\mathrm{Mo}-\mathrm{O}}$, bond valence for the average Mo - O interatomic distance (1.75 $\AA$ - see Table 3 ) also results in a sum larger than 2.0 v.u. for the oxygen atom which leads again to forbidden connections with the network formers within the glass structure. The mean of the $\mathrm{S}_{\mathrm{Mo} \text {-O }}$ bond valences for the 4-coordinated Mo cation can be used to estimate the oxidation state. Using this model, the Mo cation in the MCNB glass was found to have an oxidation state +6 , whereas Mo in the MLNB glass has a redox oxidation state, +5 . Electron spin resonance (ESR) spectra of alkali borosilicate nuclear waste glasses, containing $1 \% \mathrm{~mol}^{\mathrm{MoO}} 3$, melted under reducing conditions ${ }^{56,57}$ indicate that at least partial reduction of the oxidation state of Mo cations has occurred. Moreover, ESR studies in soda lime silicate glasses ${ }^{9,56-59}$ have shown that even under oxidising conditions some of the molybdenum is present as $\mathrm{Mo}^{3+}$ or $\mathrm{Mo}^{5+}$. 
The alkali and alkaline-earth cations have a multiple role within the borosilicate network as they can act as network modifiers ${ }^{43}$ and charge compensators. ${ }^{8}$ MD simulations in aluminoborosilicate glasses show that most of the $\mathrm{Na}$ cations charge compensate network forming $\mathrm{Al}$ or $B$ atoms. These calculations have been recently improved by a combination of MD and reverse Monte Carlo calculations based on wide angle $X$-ray scattering. ${ }^{47}$ The three cations $(\mathrm{Ca}$, $\mathrm{Na}$ and $\mathrm{Li}$ ) seems to have different coordination environments within our two simulated glasses.

In MCNB glass $\mathrm{Na}$ and $\mathrm{Ca}$ cations have a pure network modifying role. The borosilicate network is significantly modified as the cations break the bonds between the Si/B and $\mathrm{O}$ atoms and they are linked predominantly to the $\mathrm{SiO}_{4}$ tetrahedra with weak and non-directional bonds to oxygen. The cations tend to cluster within the glass structure, forming the domains in which the molybdate groups prefer to locate (see Fig. 5 and 6). In addition, B ions are mainly 3 coordinated within the MCNB glass. The crystalline molybdates will form as a result of the higher availability of modifier cations in presence of majority 3-coordinated boron, as the only charge compensating cations that can be found around the molybdate tetrahedron to stabiise its negative charge are $\mathrm{Na}$ and $\mathrm{Ca}$ cations.

In contrast, in MLNB glass the tetrahedral local environment for the $B$ ions is more favourable within the glass structure. The number of $\mathrm{Na}$ cations able to compensate $\mathrm{MoO}_{4}$ tetrahedra strongly decreases when the $\mathrm{B}_{2} \mathrm{O}_{3}$ concentration increases and the $\mathrm{Na}_{2} \mathrm{O}$ decreases. The $\mathrm{Na}$ - O bond length elongates (from 2.34 $\AA$ in MCNB to $2.39 \AA$ in MLNB) and the Na cations move from a position near non-bridging oxygen to a position near $\mathrm{BO}_{4}$ tetrahedra to act as charge compensators. Consequently, the depolymerised regions in which the $\mathrm{MoO}_{4}$ units are usually located become depleted in $\mathrm{Na}$. $\mathrm{Li}$ cations, according to their first coordination environment, seems to act as pseudo-network formers within the MLNB glass. The replacement of $\mathrm{CaO}$ for $\mathrm{Li}_{2} \mathrm{O}$ oxide leads to less depolymerisation of the borosilicate network allowing the $\mathrm{MoO}_{4}$ units to approach closer to the borosilicate network.

Structural investigations in previous experimental studies of sodium calcium borosilicate nuclear glasses containing $\mathrm{MoO}_{3}$, by MAS NMR and Raman spectroscopy, ${ }^{10,12}$ revealed that increasing the $\mathrm{B}_{2} \mathrm{O}_{3}$ concentration strongly modifies the distribution of $\mathrm{Na}$ cations within the glass network, which leads to an evolution of the nature of molybdates that can crystallise during melt cooling.

\section{Conclusions}

The Mo local environment was investigated by means of ab initio molecular dynamics simulations in two models of nuclear waste glasses with different molar compositions. Mo cations were found to exist in a tetrahedral environment forming almost perfect $\mathrm{MoO}_{4}$ tetrahedra in both glass models. The first peak in the Mo-O radial distribution function $g(r)$ is at $1.75 \AA$ for both compositions. However, in the MLNB glass, the $g(r)$ 
manifests a second sharp peak in the first coordination shell, which corresponds to a larger interatomic distance, $1.94 \AA$, indicating a qualitatively different type of Mo-O bonding. Detailed structural analysis demonstrated that Mo cations have different local atomic environment, coupled with the electronic state of the Mo species, in the MLNB and MCNB glasses. The structural data from AIMD, together with the bond valence model, indicate that the shorter Mo-O bonds has $\mathrm{a}+6$ oxidation state for the Mo cation, which leads to the crystallisation of the molybdate tetrahedron. The $\mathrm{MoO}_{4}$ in $\mathrm{MCNB}$ glass is isolated from the borosilicate network and is located in a depolymerised region of the glass structure surrounded by alkali and alkaline-earth cations. On the contrary, the longer Mo-O bonds result in a redox state ( +5 oxidation state) at room temperature for the Mo cation in MLNB glass, which leads to a connection of the $\mathrm{MoO}_{4}$ tetrahedron with the borosilicate network. Reducing the valency of the Mo cations could both eliminate the formation of the isolated alkali/alkaline-earth molybdates and increase the solubility of Mo cation in the borosilicate glass network by reducing the field strength around the cation.

Atomistic simulations are able to provide atomic pictures of the glass structures and detailed insight into compositional - atomic structure relationships. The stability of the Mo cation in the polymerised glass network seems to be correlated to the glass composition. Such understanding of how the chemical nature and molar composition of the nuclear waste glass affect the local atomic structure of Mo and its solubility within the glass network is invaluable in design of nuclear waste glasses with elevated demand for controlled segregation rate of the undesirable crystalline molybdates. Our results suggest that increasing the $\mathrm{B}_{2} \mathrm{O}_{3}$ concentration, decreasing the $\mathrm{Na}_{2} \mathrm{O}$ concentration and replacing of $\mathrm{CaO}$ for $\mathrm{Li}_{2} \mathrm{O}$ oxide will decrease the tendency to form yellow phase within the glass structure.

\section{Acknowledgements}

K.K. was supported through the Impact Studentship scheme at UCL co-funded by the IHI Corporation and UCL.

P.V.S. thanks the Royal Society, which supported preliminary work on this project, and the Laboratory Directed Research and Development program at PNNL, a multiprogram national laboratory operated by Battelle for the U.S. Department of Energy.

Via our membership of the UK's HEC Materials Chemistry Consortium, which is funded by EPSRC (EP/L000202), this work used the ARCHER UK National Supercomputing Service (http://www.archer.ac.uk). 


\section{References}

1 I. W. Donald, B. L. Metcalfe and R. N. J. Taylor, J. Mater. Sci, 1997, 32, 5851

2 M. I. Ojovan and W. E. Lee, An Introduction to Nuclear Waste Immobilisation, Elsevier, Amsterdam, 2005

3 J. A. C. Marples, Glass Technol., 1988, 29, 230

4 M. I. Ojovan and O. G. Batyukhnova, Glasses for Nuclear Waste Immobilisation, WM' 07 Conference, Tucson AZ, February 25 - March 1, 2007

5 B. Luckscheiter and M. Nesovic, Waste Management, 1996, 16, 571

6 W. Lutze and R. C. Ewing, Radioactive Wasteforms for the Future, North Holland, Amsterdam, 1988

7 R. J. Short, R. J. Hand, N. C. Hyatt, and G. J. Möbus, Nucl. Mater., 2005, 340, 179

8 G. Calas, M. Le Grand, L. Galoisy and D. Ghaleb, J. Nucl. Mater., 2003, 322, 15

9 F. Farges, R. Siewert, G. E. Brown, A. Guesdon and G. Morin, Can. Mineral., 2006, 44, 731

10 D. Caurant, O. Majérus, E. Fadel, A. Quintas, C. Cervais, T. Charpentier and D. Neuville, J. Nucl. Mater., 2010, 396, 94

11 A. Paul, J. Non-Cryst. Solids, 1990, 123, 354

12 D. Caurant, O. Majérus, E. Fadel, M. Lenoir, C. Cervais and O. Pinet, J. Am. Ceram. Soc., 2007, 90, 774

13 A. Tilocca and N. H. de Leeuw, J. Mater. Chem., 2006, 16, 1950

14 K. Trachenko, V. V. Brazhkin, G. Ferlat, M. T. Dove and E. Artacho, Phys. Rev. B, 2008, 78, 172102

15 A. Pedone, G. Malavasi, M. C. Menziani, A. N. Cormack and U. Serge, J. Phys. Chem. B, 2006, 110, 11780

16 J. Sarnthein, A. Pasquarello and R. Car, Phys. Rev. Lett., 1995, 74, 4682

17 R. M. Van Ginhoven, H. Jonsson and L. R. Corrales, Phys. Rev. B, 2005, 71, 024208

18 S. Ispas, M. Benoit, P. Jund and R. Jullien, Phys. Rev. B, 2001, 64, 214206

19 S. Ispas, M. Benoit, P. Jund and R. Jullien, J. Non-Cryst. Solids, 2002, 307, 946

20 D. Donadio, M. Bernasconi and F. Tassone, Phys. Rev. B, 2004, 70, 214205

21 L. Pedesseau, S. Ispas and W. Kob, Phys. Rev. B, 2015, 91,134202

22 A. Tilocca and N. H. de Leeuw, J. Phys. Chem. B, 2006, 110, 25810

23 A. Tilocca, Phys. Rev. B, 2007, 76, 224202

24 A. Tilocca, J. Chem. Phys., 2010, 133, 014701

25 J. K. Christie, R. I. Ainsworth and N. H. de Leeuw, Biomaterials, 2014, 35, 6164

26 W. Smith and T. R. Forester, J. Mol. Graph., 1996, 14, 136

27 J. E. Lennard-Jones, The Proceedings of the Physical Society, 1931, 43, 461

28 A. T. Hagler, S. Lifson and P. Dauber-Osguthorpe, J. Am. Chem. Soc., 1979, 101, 5122

29 S. Lifson, A. T. Hagler and P. Dauber-Osguthorpe, J. Am. Chem. Soc., 1979, 101, 5131

30 S. Nosé, Molecular Physics, 1984, 52, 255

31 S. Nosé, J. Chem. Phys., 1984, 81, 511

32 W. G. Hoover, Phys. Rev. A, 1985, 31, 1695

33 G. Bussi, D. Donadio and M. Parrinello, J. Chem. Phys., 2007, 126, 014101

34 F. Michel, L. Cormier, P. Lombard, B. Beuneu, L. Galoisy and G. Calas, J. Non-Cryst. Solids, 2013, 379, 169

35 J. M. Roderick, D. Holland, A. P. Howes, C. R. Scales, J. Non-Cryst. Solids, 2001, 293-95, 746 
36 J. VandeVondele, M. Krack, F. Mohamed, M. Parrinello, T. Chassaing and J. Hutter, Comp. Phys. Comm., 2005, 167, 103

37 W. Kohn and L. J. Sham, Phys. Rev. Lett., 1965, 140, A1133

38 J. P. Perdew, K. Burke and M. Ernzerhof, Phys. Rev. Lett., 1996, 77, 3865

39 J. VandeVondele and J. Hutter, J. Chem. Phys., 2007, 127, 114105

40 S. Goedecker, M. Teter and J. Hutter, Phys. Rev. B, 1996, 54, 1703

41 J. K. Christie and A. Tilocca, Adv. Eng. Mater., 2010, 12, 326

42 J. K. Christie, A. Pedone, M. C. Menziani and Tilocca, J. Phys. Chem. B, 2011, 115, 2038

43 W. H. Zachariesen, J. Chem. Soc., 1932, 54, 3841

44 X. Yuan and A. N. Cormack, J. Non-Cryst. Solids, 2001, 283, 69

45 A. N. Cormack and J. Du, J. Non-Cryst. Solids, 2001, 293-95, 283

46 J. Du and A. N. Cormack, J. Non-Cryst. Solids, 2004, 283, 69

47 L. Cormier, D. Ghaleb, J. M. Delaye and G. Calas, Phys. Rev. B, 2000, 61, 14495

48 O. Majérus, L. Cormier, G. Calas and B. Beuneu, Phys. Rev. B, 2003, 67, 024210

49 A. J. Connelly, K. P. Travis, R. J. Hand, N. C. Hyatt and E. Maddrell, J. Am. Ceram. Soc., 2011, 94, 151

50 J. Zhao, P. H. Gaskell, M. M. Cluckie and A. K. Soper, J. Non-Cryst. Solids, 1998, 232-34, 721

51 I. D. Brown and R. D. Shannon, Acta Crystallogr. A, 1973, A29, 266

52 I. D. Brown and D. Altermatt, Acta Crystallogr. B, 1985, 41, 240

53 I. D. Brown and D. Altermatt, Acta Crystallogr. B, 1985, 41, 244

54 L. Galoisy, L. Cormier, S. Rossano, A. Ramos, G. Calas, P. Gaskell and M. Le Grand, Mineral. Mag., 2000, 64, 207

55 L. Pauling, J. Am. Chem. Soc., 1929, 51, 1010

56 B. Camara, W. Lutze and J. Lux, Sci. Basis for Nucl. Waste Man., 1980, 2, 93

57 A. Horneber, B. Camara and W. Lutze, Sci. Basis for Nucl. Waste Man., 1982, 5, 279

58 R. J. Landry, J. Chem. Phys., 1968, 48, 1422

59 V. Aubin-Chevaldonnet, D. Gourier, D. Caurant, S. Esnouf, T. Charpentier and J. M. Costantini, J. Phys. Condens. Mattter, 2006, 18, 4007 